\title{
The nature and nurture of star clusters
}

\author{
Bruce G. Elmegreen \\ IBM Research Division, T. J. Watson Research Center, 1101 Kitchawan Road, \\ Yorktown Heights, NY 10598 USA \\ email: bge@us.ibm.com
}

\begin{abstract}
Star clusters have hierarchical patterns in space and time, suggesting formation processes in the densest regions of a turbulent interstellar medium. Clusters also have hierarchical substructure when they are young, which makes them all look like the inner mixed parts of a pervasive stellar hierarchy. Young field stars share this distribution, presumably because some of them came from dissolved clusters and others formed in a dispersed fashion in the same gas. The fraction of star formation that ends up in clusters is apparently not constant, but may increase with interstellar pressure. Hierarchical structure explains why stars form in clusters and why many of these clusters are self-bound. It also explains the cluster mass function. Halo globular clusters share many properties of disk clusters, including what appears to be an upper cluster cutoff mass. However, halo globulars are self-enriched and often connected with dwarf galaxy streams. The mass function of halo globulars could have initially been like the power-law mass function of disk clusters, but the halo globulars have lost their low-mass members. The reasons for this loss are not understood. It could have happened slowly over time as a result of cluster evaporation, or it could have happened early after cluster formation as a result of gas loss. The latter model explains best the observation that the globular cluster mass function has no radial gradient in galaxies.
\end{abstract}

Keywords. open clusters and associations: general, solar neighborhood, galaxies: star clusters, stars: formation

\section{Introduction}

Star formation is hierarchical in time and space, suggesting that turbulent processes partition the gas. Star formation is also clustered to varying degrees, suggesting the action of some dimensionless quantity such as the turbulent Mach number. Here, we review recent observations of clusters and associations with emphasis on hierarchical structure for the young regions, the resulting mass functions for open and globular clusters, and the likely dwarf galaxy origin for some halo globular clusters in the Milky Way.

\section{Hierarchical structure in young clusters}

\subsection{Spatial correlations}

Piskunov et al. (2006) identified several 'open cluster complexes' according to groupings of position, velocity, and age of clusters in the solar neighborhood (see also Lyngå 1982; Kharchenko et al. 2005). Examples of these complexes included one of intermediate age consisting of seven clusters in Perseus-Auriga, an older one with six clusters in the Hyades, and a younger one with 23 members that is essentially Gould's Belt. A typical complex spans a distance of several hundred parsecs. They are probably the same objects that were called 'star complexes' by Efremov (1995), who found them as groupings of Cepheid variables and red supergiants. Ivanov (2005) catalogued star complexes in M33 based on clusterings of blue stars, HiI regions, and Wolf-Rayet stars. 
De la Fuente Marcos \& de la Fuente Marcos (2008) identified five open cluster complexes from cluster positions and velocities within $2.5 \mathrm{kpc}$ of the Sun: at a Galactic longitude of $l=12^{\circ}$ and a distance of $1300 \mathrm{pc}$, there is the Scutum-Sagittarius complex; at $l=75^{\circ}$ and $1400 \mathrm{pc}$, the Cygnus complex; at $l=132^{\circ}$ and $2000 \mathrm{pc}$, the CassiopeiaPerseus complex; at $l=200^{\circ}$ and $500 \mathrm{pc}$, the Orion complex, and at $l=295^{\circ}$ and 2000 pc, the Centaurus-Carina complex. They suggest that open cluster complexes are fragments from a common gas cloud. This is the usual explanation for star complexes too.

The gas clouds that form star complexes contain $\sim 10^{7} \mathrm{M}_{\odot}$ of $\mathrm{HI}$ or $\mathrm{H}_{2}$, and are fragmented into giant molecular clouds (GMCs) and molecular cloud cores that form OB associations and OB subgroups or clusters, respectively (e.g., Elmegreen 2007). These enormous regions usually occur in galactic spiral arms with a separation of several kiloparsecs (McGee \& Milton 1964; Elmegreen \& Elmegreen 1987). Presumably, the largest clouds are formed by galactic-scale processes (i.e., their mass is the Jeans mass of the ambient interstellar medium; ISM), and then fragment into GMCs, GMC clumps, and eventually star-forming cores. Most GMCs in the Milky Way (Grabelski et al. 1987) and M33 (Engargiola et al. 2003) are found in these gas giants, which are primarily composed of HI. In M51, where the gas is more molecular overall because of the higher pressure, the gas giants are mostly molecular (Rand \& Kulkarni 1990).

Not all star formation produces dense clusters. Elias et al. (2009) showed that clustering varies spatially in Gould's Belt, with a larger fraction of stars ending up in clusters in Orion than in the Sco-Cen region. This gradient in cluster fraction corresponds to a gradient in young cluster density, suggesting that the dense, high-pressure centers of star complexes produce stars more efficiently than low-pressure peripheral regions. Higher efficiency means that the final ratio of star-to-gas mass is higher, implying that the region is more likely to end up self-bound as a cluster after the gas leaves.

Hierarchical structure extends from star complexes to embedded clusters to individual young stars inside embedded clusters. Feitzinger et al. $(1984,1987)$ were among the first to recognize hierarchical or fractal structure in large-scale star-forming regions. An important point about hierarchical structure is that the average density of gas increases down the hierarchy, i.e., towards smaller fragment masses. The mass fraction represented by dense star-forming clumps increases along this sequence too. Bound regions require a high mass fraction for stars and so appear only at the bottom of the hierarchy. This is the primary reason why bound clusters are dense (much denser than the background tidal limit, which is all they would need for self-boundedness at zero velocity dispersion). With hierarchical structure, the average gas density is high in regions where the star-formation efficiency is high. This is the reason why most star formation begins in the form of a dense embedded cluster (Elmegreen 2008).

Scheepmaker et al. (2009) studied clusters in M51. Autocorrelation functions for these clusters in three age bins show that the youngest sample is well correlated: it is hierarchical with a fractal dimension of $\sim 1.6$. The autocorrelation means that clusters are distributed in cluster pairs and triplets, and these are inside clusters complexes and so on up to scales greater than $1 \mathrm{kpc}$. Clusters in the Antennae galaxy are also autocorrelated up to $\sim 1 \mathrm{kpc}$ (Zhang et al. 2001). Sánchez \& Alfaro (2008) studied HiI regions in a number of galaxies. They found that the positions of the brightest HiI region have the smallest fractal dimensions, which means they are still hierarchical. For the galaxy NGC 6946, the high-brightness Hiı regions have a fractal dimension $D_{\mathrm{c}}=1.64$, those of medium brightness have $D_{\mathrm{c}}=1.82$, and those with low brightness have $D_{\mathrm{c}}=1.79$. Sánchez \& Alfaro (2008) also found that $D_{\text {c }}$ decreases slightly with galaxy brightness, which means that low-luminosity galaxies have more hierarchical structure in the 
positions of their Hir regions. Presumably these low-luminosity galaxies have less shear to smooth out the hierarchical birth positions of the HiI regions.

Elmegreen et al. (2006) studied the size distribution of star-forming regions in NGC 628 by box counting, which is a common technique for measuring fractal dimensions. They used a high-resolution image of this galaxy from the ACS instrument on HST and blurred it in successive stages. The number of regions at each blurring stage was counted with SExTRACTOR. They found that the cumulative size distribution of starforming regions is $n(R) \mathrm{d} R \sim R^{-2.5} \mathrm{~d} R$ for size $R$, and they fit this to a projected fractal Brownian-motion density distribution having a $3 \mathrm{D}$ power spectrum with the Kolmogorov slope, -3.66. Elmegreen et al. (2003) measured azimuthal intensity profiles of optical light from whole galaxies and found that they also have power-law power spectra like turbulence. For example, the young stars and dust clouds seen in optical images of the flocculent galaxy NGC 5055 have the same scale-free distribution as the Hi gas in the Large Magellanic Cloud (LMC). Both give a Kolmogorov power spectrum. Block et al. (2009) presented power spectra of Spitzer Space Telescope images of several galaxies, using the near-infrared passbands. They found that the passbands dominated by stars (channels 1 and 2) had power spectra from noise plus the point-spread function that comes from the unresolved stellar images, while the passbands dominated by dust and polycyclic aromatic hydrocarbon emission (channel 4) had Kolmogorov power spectra, clearly different in slope from the star-dominated power spectra. Images of the range of Fourier components that give the power-law power spectrum in channel 4 show the resolved hierarchical part of the galaxy.

\subsection{Time correlations}

Clusters are also correlated in time in the sense that the age difference increases with separation. Efremov \& Elmegreen (1998) found this for $\sim 600$ clusters in the LMC, and de la Fuente Marcos \& de la Fuente Marcos (2009a) found it for local Milky Way clusters. The slope of the correlation is about 0.5. This is interpreted as an indication that the duration of star formation in a complex increases as the square root of the size (Elmegreen \& Efremov 1996). Such a relation between time and size also applies to self-gravitating clouds with a common pressure: self-gravity means that $G M / R \sim 5 V^{2}$, and a common pressure $P$ means that $0.1 G M^{2} / R^{4} \sim P$ (for mass $M$, radius $R$, and velocity dispersion $V)$. Eliminating $M$ gives $V^{2} / R=(0.4 P G)^{0.2}$, which is constant. As a result, the crossing time is $R / V=R^{0.5}(0.4 P G)^{-0.25}$. This is the same form as the observed relation between star-formation duration and region size. For typical $P=10^{6} k_{\mathrm{B}}, R / V=2.3 \times 10^{4} R^{0.5}$ in cgs units, or $R / V=1.3 R^{0.5} \mathrm{Myr}$ for $R$ in pc and $V$ in $\mathrm{km} \mathrm{s}^{-1}$. Efremov \& Elmegreen (1998) found, for an age range of 1-100 Myr in the LMC, that the time separation equals $30 \mathrm{Myr}$ times the spatial separation in degrees to the power 0.33 (for the LMC, 1 degree $=$ $960 \mathrm{pc}$ ). This is about the same relation as in the above simple derivation. For the Milky Way, de la Fuente Marcos \& de la Fuente Marcos (2009a) found a time separation equal to 11.1 Myr times the spatial separation in parsecs to the power 0.16. Both the LMC and Milky Way exhibit good correlations, but the slopes are different. De la Fuente Marcos \& de la Fuente Marcos (2009a) suggest that if cluster disruption is taken into account, the slopes are a bit steeper, between $\sim 0.3$ for the Milky Way and $\sim 0.5$ for the LMC. If clusters within $2 \mathrm{kpc}$ of the Sun are considered, the local slope could be $\sim 0.4$.

\subsection{Cluster pairs}

The time-distance correlation among young clusters implies that there should be pairs of clusters born at about the same time and place. Indeed, such pairs are well observed. They were originally discovered in the LMC by Bhatia \& Hatzidimitriou (1988) and in 
the Small Magellanic Cloud (SMC) by Hatzidimitriou \& Bhatia (1990). Dieball et al. (2002) studied them again in the LMC. De la Fuente Marcos \& de la Fuente Marcos (2009b) studied cluster pairs in the Milky Way and assessed whether they are interacting. For example, they found that NGC 3293 and NGC 3324 near $\eta$ Carinae are weakly interacting; their age difference is 4.7 Myr. Similarly, NGC 659 and NGC 663 are weakly interacting and their age difference is 19.1 Myr. Cluster pairs are the closest members of the hierarchy of clusters found by autocorrelation studies, power spectra, fractal analysis, and box-counting techniques.

\subsection{Individual stars}

Individual young stars are correlated even if they are not in clusters. The two-point correlation function for stars in Taurus has a power-law form (Gomez et al. 1993; Larson 1995). Bastian et al. (2009) looked at the positions and ages of stars in the LMC. They included 2000 sources in each of several age intervals in the color-magnitude diagram. The two-point correlation function for these field stars has a slope that gradually changes from -0.5 at the youngest age to 0 at an age of $175 \mathrm{Myr}$. The coefficient in the twopoint correlation function goes systematically to zero along this age sequence too. They also studied the Cartwright \& Whitworth (2004) $Q$ parameter, which is the ratio of the average minimum-spanning-tree length to the average correlation length. When a distribution of objects is fragmented into pieces, $Q$ is less than 0.8 or so; when it is smooth or with a smooth gradient, $Q$ is closer to 1 . Bastian et al. (2009) found that $Q$ also shows a gradual variation with stellar age, from $\sim 0.55$ to $\sim 0.75$ as age increases to $175 \mathrm{Myr}$. This is consistent with the trend in the two-point correlation function: older field stars are less clumped. Gieles et al. (2008) did the same analysis for SMC stars, finding the same age trends in the two-point correlation function and $Q$ out to about 100 Myr.

Odekon (2006) determined a 'correlation dimension,' $d_{\mathrm{c}}$, for stars seen by HST in dwarf galaxies. The correlation dimension comes from the equation $N(r) \propto r^{d_{c}}$, where $N(r)$ is the average number of stars in a region of radius $r$. She found that the brightest dwarf has the largest $d_{\mathrm{c}}$ and the faintest dwarf has the smallest $d_{\mathrm{c}}$. This is consistent with the trend for HiI-region clustering in galaxies found by Sánchez \& Alfaro (2008).

\subsection{Internal cluster structure}

The hierarchy of young stellar structure often persists even inside currently forming clusters. Testi et al. (2000) found substructure in an embedded infrared cluster in Serpens, Smith et al. (2005) found it in the $\rho$ Oph region, and Dahm \& Simon (2005) found four subclusters with $\sim$ Myr age differences in NGC 2264. Azimuthal profiles of young clusters have non-Poissonian distributions as well (Gutermuth et al. 2005). A recent X-ray map of young stars in NGC 6334 (Feigelson et al. 2009) shows substantial substructure in the positions of soft and hard X-ray sources (which correspond to embedded populations with less than and greater than 10 magnitudes of visual extinction, respectively). The X-ray maps are nearly complete for stars more massive than the Sun. Sánchez \& Alfaro (2009) show that for 16 Milky Way clusters, the stars in the younger, bigger clusters are more clumped. Evidently, as a cluster ages, its subclusters mix and the substructure smooths out.

Schmeja et al. (2008) studied clumpy structure in four embedded clusters. For IC 348, NGC 1333, and the $\rho$ Oph region, $Q$ is lower (more clumpy) for class $0 / 1$ objects (young) than for class $2 / 3$ (old). Also, among four subclumps in $\rho$ Oph, $Q$ is lower and it is more gaseous where class $0 / 1$ objects dominate, and $Q$ is also lower for class $0 / 1$ alone than for class $2 / 3$. For an even younger stage, Johnstone et al. (2000, 2001), Enoch et al. (2006), 
and Young et al. (2006) found that the millimeter-wave pre-stellar clumps in several regions are spatially correlated.

\subsection{Summmary: 'clusters' are the dense cores of a pervasive hierarchy of star formation}

Hierarchal structure in the ISM presumably comes from self-gravity and turbulence. This gas structure continues to substellar scales, as shown by high-resolution molecular observations. The densest regions, which are where individual stars form, are often clustered into the next-denser regions. Stars form in the densest regions, at first somewhat independently it seems, and then they move around, possibly interact, and ultimately mix together inside the next-lower-density region. That mixture is the 'cluster.' More and more subclusters mix over time until the cloud disrupts. The net efficiency of star formation (fractional star mass) is automatically high on small and dense scales because of the hierarchy of structures.

Clusters are the inner mixed parts of the hierarchy of young stellar structures. The hierarchy that is present on larger scales was also present on smaller scales before this mixing. If we consider a cluster's 'Nature' to be its internal properties, and its 'Nurture' to be its external properties, then Nature and Nurture for a star cluster are essentially the same thing. Internal and external depend on the extent to which gravitational mixing has occurred at the time of observation.

\section{Unclustered star formation}

Barbá et al. (2009) studied the giant star-forming region NGC 604 in M33 with HST/NICMOS to look for obscured young clusters. They found that it contains almost no clusters but that most star formation is distributed, in agreement with Hunter et al. (1996). Giant molecular clouds also show some star formation in a distributed form (Megeath et al. 2004; Jørgensen et al. 2006, 2007), although in the solar neighborhood, most star formation is clustered. Maíz-Apellániz (2001) generalized the discussion by considering three types of clustering, (i) compact clusters with weak unclustered halos typically measuring $50 \times 50 \mathrm{pc}^{2}$, (ii) compact clusters with strong halos, measuring $100 \times 100 \mathrm{pc}^{2}$, and (iii) purely hierarchical star formation with no clusters.

A possible reason for variations in young stellar clustering is explored by Elmegreen (2008). The basic point was outlined above, that hierarchical star formation automatically leads to high efficiencies and bound stellar clustering at high densities. The point now is to find the mass fraction of star formation that has a sufficiently high efficiency to produce a bound cluster after the gas leaves. This fraction can be calculated from the density probability density function (pdf) in a GMC. The density pdf has a log-normal form in isothermal turbulence and a power-law form at high density when self-gravity is important (Klessen 2001). An integral over density times the density pdf gives the mass. The integral illustrates the importance of the breadth of the density pdf: for a narrow pdf (i.e., with a low dispersion), the critical efficiency is reached at a high density and a low mass fraction, while for a wide pdf, the critical efficiency is reached at a low density and a high mass fraction. What matters is the slope of the pdf at the density where the efficiency becomes critical for producing a bound cluster. Thus the breadth of the pdf enters into this slope. Also entering is the centroid density. Generally, the pdf is in the high-density falling part at the critical value for high efficiency. Then a shift in the pdf towards higher density will also make the slope shallower there (for the lognormal case). Thus, either a broader pdf or a higher average density will lead to a higher fraction of the star-formation mass in the form of bound clusters. A broader pdf could arise from a 
higher turbulent Mach number. It follows that higher-pressure regions are more likely to form bound clusters.

\section{Cluster mass functions}

The mass function for bound clusters is approximately $n(M) \mathrm{d} M \propto M^{-2} \mathrm{~d} M$ for a given age range (Elmegreen \& Efremov 1997; Zhang \& Fall 1999; de Grijs et al. 2003; de Grijs \& Anders 2006). This function is also observed for various ages, although the lower limit to the power law depends on the age because of cluster-magnitude detection limits and cluster fading with age (Elmegreen \& Efremov 1997; Hunter et al. 2003; de Grijs \& Anders 2006).

The cluster mass function resembles the mass function of cluster-forming cloud clumps (Reid \& Wilson 2005; Rathborne et al. 2006), and both presumably get their form from the hierarchical structure of interstellar matter. In a hierarchy, all of the mass is represented at all levels, so the total mass in each level (which has logarithmic intervals of mass), $M n(\log M) \mathrm{d} \log M$, is the same as the total mass in any other level. It follows that $M n(\log M) \mathrm{d} \log M=$ constant $\times \mathrm{d} \log M, n(\log M) \propto M^{-1}$, and $n(M) \propto M^{-2}$. Similarly, if subclouds are randomly selected from a hierarchical cloud, their distribution function will be $M^{-2}$ too. Or, if we generate a fractal Brownian-motion cloud, the mass distribution of clumps will be close to $M^{-2}$, with a preferred value of the power spectrum given by the Kolmogorov-turbulence law (Elmegreen et al. 2006).

Recent observations suggest that the power-law mass function sometimes has an upper mass cutoff, making it a Schechter function (as originally applied to galaxies; Schechter 1976). These cutoffs have been observed in M51 (Gieles et al. 2006a,b) and several other galaxies (Waters et al. 2006; Larsen 2009).

Before we turn to halo globular clusters, the essential points of the preceding sections can be summarized as follows:

- Gas is hierarchical in space and time, presumably as a result of turbulence and self-gravity.

- Therefore star formation is hierarchical.

- Therefore the efficiency of star formation $\left(M_{\text {stars }} / M_{\text {total }}\right)$ increases with average density.

- Therefore bound objects (which require high efficiency) form at high density. The result is a cluster.

- Finally, the mass function of all this structure is a power law with a slope of -2 for equal intervals of mass.

- The origin of the upper cutoff mass for clusters is unexplained.

\section{Globular clusters}

The mass functions of galactic clusters, reviewed above, resemble the upper parts of the mass functions of globular clusters, which appear to have lost their low-mass members. Jordán et al. (2007) fit power laws to the upper parts of the globular cluster mass functions in Virgo galaxies $\left(3-20 \times 10^{5} \mathrm{M}_{\odot}\right.$ clusters $)$. They found that the upper-mass power law is steeper for lower-luminosity galaxies, and the width of the lognormal mass function is also smaller for lower-luminosity galaxies. This result implies that low-mass galaxies have a lower upper-mass cutoff for their clusters. Waters et al. (2006) measured the mass function of M87 globular clusters with HST and also found that the best fit was an evolved Schechter function with a cutoff mass of $M_{\mathrm{c}}=10^{6.4} \mathrm{M}_{\odot}$. The evolution models in Waters et al. assumed various mass dependencies for cluster evaporation, with 
the best fit having no mass dependence. For either this standard model of mass-loss rate, $\mathrm{d} M / \mathrm{d} t=$ constant, or the Lamers et al. (2006) model, $\mathrm{d} M / \mathrm{d} t \propto M^{0.38}$, the low-mass globular clusters evaporate first, leaving only the high-mass clusters after a Hubble time.

The problem with evaporation models is that globular cluster mass functions are independent of galactocentric radius in galaxies (Kundu et al. 1999; Jordán et al. 2007), while the evaporation rate depends on the tidal density, which depends on galactocentric radius. Thus, the outer regions of galaxies should have more low-mass globular clusters remaining than the inner regions, but they do not. One proposed solution is that globular cluster orbits are highly radial (Fall \& Zhang 2001), but this disagrees with the observed profile of globular cluster velocity dispersion versus radius (Vesperini et al. 2003).

McLaughlin \& Fall (2008) separated the Milky Way globular cluster luminosity function into three groups according to the density at half-light radius. The peak mass was found to depend on the density as expected for $\mathrm{d} M / \mathrm{d} t=$ constant evaporation of a Schechter function, and the mass functions are independent of position, as required. Chandar et al. (2007) found the same for M104. However, low-density globular clusters are of lower mass anyway because they all have about the same radius (e.g., McLaughlin 2000). Perhaps density-dependent evaporation is the reason they all have the same radius, but this has not been demonstrated independently.

Another option is that the globular cluster mass function was peaked from a young age (Vesperini 2000; Parmentier \& Gilmore 2007) and has not evolved much since then. Evaporation tends to preserve a peak in the mass function once it forms (Vesperini 1998). Parmentier et al. (2008) showed that cluster disruption during initial gas removal can convert a power-law mass function into a lognormal mass function at a very early stage. This would satisfy the observations that show no radial gradients in the mass function. The problem with this model is that modern clusters do not lose their initial power-law mass functions so quickly. The difference could be that halo globular clusters formed with a lower efficiency $(<25 \%)$ than disk clusters $(\sim 40 \%)$ because cluster disruption is more likely during gas loss for low efficiencies. We note that, in the hierarchical model of cluster formation, lower efficiency for a given cluster density (required to define an object as a cluster) follows from lower ISM pressures (Elmegreen 2008), and that is consistent with globular cluster formation in dwarf galaxies.

Halo globular clusters differ from disk open clusters in several ways. Globulars are more massive and this presumably allows self-enrichment of heavy elements from redgiant winds. Globular clusters are also older, and this means that evolutionary effects are more prominent, such as evaporation and core collapse. Globular clusters are lower in metallicity than disk clusters, and this means that stellar winds are weaker and any self-enrichment is more obvious. Globular clusters are also in the halo and many appear to come from dissolved companion dwarf galaxies. Others could come from the starburst phase of a merger when the disk and its clusters are scattering into the halo. Yet others could come from star formation in small galaxies before the large galaxies formed.

Harris (2009) used the HST to observe six giant-elliptical galaxies within $40 \mathrm{Mpc}$. He found 7800 globular clusters with half-light radii larger than $1.5 \mathrm{pc}$. The blue (low metallicity) and red (high metallicity) sequences found previously for many globular cluster systems are clear in these new data. The blue sequence turns slightly redder at high mass, suggesting self-enrichment for $M>10^{6} \mathrm{M}_{\odot}$. Normal blue globulars have $[\mathrm{Fe} / \mathrm{H}] \sim-1.5$ dex, while in his survey, the most massive blue globulars have $[\mathrm{Fe} / \mathrm{H}] \sim-1$ dex. This redward trend toward higher mass among the blue globulars was also found in M87 by Peng (2009). Bailin \& Harris (2009) suggest supernova ejecta are trapped in the more massive clusters during the gas-rich formation phase. Similar observations are found in Harris et al. (2006), Strader et al. (2006), and Mieske et al. (2006). 
Bedin et al. (2004) found multiple main sequences and main-sequence turnoffs in $\omega$ Cen. The multiple main sequence implies there is a range of He abundances (D'Antona et al. 2002; Norris 2004), while the multiple subgiant branches mean there is a range of ages (Milone et al. 2008) or CNO abundances (Cassisi et al. 2008). Self-enrichment is likely. Ventura et al. (2009) found a split subgiant branch in NGC 1851. They modeled the second generation with a CNO abundance three times that of the first generation. They suggested that massive asymptotic giant branch stars in the first generation have ejecta with five times the CNO abundances of the stars themselves, and these ejecta were diluted by $50 \%$ with pristine gas to keep the He abundance low. CNO enrichment is usually so large in globular clusters that the progenitor stars have to outnumber the cluster stars. Either the former cluster was much more massive (D'Ercole et al. 2008) or the globular cluster was the core of a dwarf galaxy which collected ejecta from many other clusters (Bekki \& Norris 2006).

Marcolini (2007) ran hydrodynamic models of star formation and metal production in a dwarf-spheroidal galaxy with an $\omega$ Cen-type cluster forming in the nucleus. They assumed star formation had a high rate for $1 \mathrm{Gyr}$ and then a lower rate for another 0.6 Gyr. The $\alpha$ elements form from Type II supernovae (SNII) quickly and Fe forms from Type Ia SNe more slowly. The SNII push out and mix with the nuclear gas, producing a uniform and growing Fe abundance. This gas resettles to the center when the massive stars are gone. SNIa then become important, and they pollute the central region locally, leading to pockets of low $\alpha / \mathrm{Fe}$. The result is a wide range of $[\mathrm{Fe} / \mathrm{H}]$ in globular cluster stars, with high $\alpha / \mathrm{Fe}$ ratios at low $[\mathrm{Fe} / \mathrm{H}]$, and an increasing dispersion towards low $\alpha / \mathrm{Fe}$ at high $[\mathrm{Fe} / \mathrm{H}]$ abundances, as observed.

Another possibility is that small clusters merge in the nucleus of a dwarf galaxy. Georgiev et al. (2009) found two globular clusters in the dwarf irregular galaxy UGCA and thought they should merge in 0.4 Gyr through dynamical friction and form a nuclear globular cluster.

The Sagittarius (Sgr) dwarf galaxy is a good example of a source for Milky Way globular clusters, having Terzan 7, Terzan 8, Arp 2, and M54 as part of its orbital debris. Carraro et al. (2007) suggested that the globular cluster Whiting 1 is also likely associated with the Sgr dwarf, which would make six in total. The age of Whiting 1 is $6.5 \pm$ $0.7 \mathrm{Gyr}$ an $[\mathrm{Fe} / \mathrm{H}]=-0.4$ to $-1.1 \mathrm{dex}$, which are consistent with the age-metallicity pattern in the Sgr dwarf. Whiting 1 also has an extended luminosity profile, presumably a tidal tail, and its position, distance, and velocity place it in the Sgr stream.

Casetti-Dinescu et al. (2009) considered the Virgo stellar stream and integrated its stellar orbits back for 5 Gyr. They found that the globular cluster NGC 2419 could be in this stream. Gao et al. (2007) found common streams for many Milky Way globular clusters, based on common energy, angular momentum, and orbital poles. Gao et al. suggest that $20 \%$ of globular clusters are in common streams.

Smith et al. (2009) noted that four globular clusters lie in a halo stellar kinematic overdensity. Their metallicities are $[\mathrm{Fe} / \mathrm{H}]=-2.22,-1.54,-1.58$ and -1.65 dex; one of them, NGC 5466, is disrupting (Odenkirchen \& Grebel 2004; Belokurov et al. 2006). Other related studies are by Dinescu et al. (1999), Palma et al. (2002), and Mackey \& Gilmore (2004).

\section{Summary}

Stars form from gas in hierarchical patterns. Clusters are the inner mixed part of this star-formation distribution. In such a hierarchy, the star-formation efficiency is automatically high on small scales, and if it is high enough, the cluster remains bound after the 
gas leaves. The cluster mass function, $\mathrm{d} N / \mathrm{d} M \sim M^{-2}$, follows from the hierarchy too. There is a possible upper cutoff mass, $M_{\mathrm{c}}$, making the mass function something like $M^{-2} e^{-M / M_{c}}$. Halo globular clusters may have a similar upper-mass cutoff and they all drop towards lower mass, without the power law. It is not yet understood if the missing low-mass clusters were removed by a long-term process of evaporation, or if they were destroyed early on by some other process, including gas loss after star formation. Globular clusters often show multiple star-formation events, which implies self-enrichment over a period of 0.1 to 1 Gyr. Perhaps their high mass and low metallicity is enough to produce this enrichment, which is not generally observed for low-mass clusters today. Or perhaps globular clusters were dwarf galaxy nuclei that collected independent clusters with a wide range of metallicities. Many Milky Way globular clusters orbit in the stellar debris streams from former dwarf galaxy companions.

\section{References}

Bailin, J. \& Harris, W. E. 2009, ApJ, 695, 1082

Barbá, R. H., Maíz Apellániz. J., Pérez, E., Rubio, M., Bolatto, A., Fariña, C., Bosch, G., \& Walborn, N. R. 2009, in: E. Pérez, R. de Grijs, \& R. M. González Delgado, Young massive star clusters: initial conditions and environments, ApSS, 324, 309, Dordrecht: Springer

Bastian, N., Gieles, M., Ercolano, B., \& Gutermuth, R. 2009, MNRAS, 392, 868

Bedin, L. R. 2004, ApJ (Letters), 605, L125

Bekki, K. \& Norris, J. E. 2006, ApJ (Letters), 637, L109

Belokurov, V., Evans, N. W., Irwin, M. J., Hewett, P. C., \& Wilkinson, M. I., 2006, ApJ (Letters), 637, L29

Bhatia, R. K. \& Hatzidimitriou, D. 1988, MNRAS, 230, 215

Block, D. L., Puerari, I., Elmegreen, B. G., Elmegreen, D. M., Fazio, G. G., \& Gehrz, R. D. 2009, ApJ, 694, 115

Carraro, G., Zinn, R., \& Moni Bidin, C. 2007, A\& $A$, 466, 181

Cartwright, A. \& Whitworth, A. P., 2004, MNRAS, 348, 589

Casetti-Dinescu, D. I., Girard, T. M., Majewski, S. R., Vivas, A. K., Wilhelm, R., Carlin, J. L., Beers, T. C., \& van Altena, W. F. 2009, ApJ (Letters), 701, L29

Cassisi, S., Salaris, M., Pietrinferni, A., Piotto, G., Milone, A. P., Bedin, L. R., \& Anderson, J. 2008, ApJ (Letters), 672, L115

Chandar, R., Fall, S. M., \& McLaughlin, D. E. 2007, ApJ (Letters), 668, L119

Dahm, S. E. \& Simon, T. 2005, AJ, 129, 829

D'Antona, F., Caloi, V., Montalbán, J., Ventura, P., \& Gratton, R. 2002, A 6 A, 395, 69

D'Ercole, A., Vesperini, E., D'Antona, F., McMillan, S. L. W., \& Recchi, S. 2008, MNRAS, 391, 825

de Grijs, R., Anders, P., Bastian, N., Lynds, R., Lamers, H. J. G. L. M., \& O'Neil Jr., E. J. 2003, MNRAS, 343, 1285

de Grijs, R. \& Anders, P. 2006, MNRAS, 366, 295

de la Fuente Marcos, R. \& de la Fuente Marcos, C. 2008, ApJ, 672, 342

de la Fuente Marcos, R. \& de la Fuente Marcos, C. 2009a, ApJ, 700, 436

de la Fuente Marcos, R. \& de la Fuente Marcos, C. 2009b, $A \mathscr{E} A$ (Letters), 500, L13

Dieball, A., Müller, H., \& Grebel, E. K. 2002, A\& $A$, 391, 547

Dinescu, D. I., Girard, T. M., \& van Altena, W. F., 1999, AJ, 117, 1792

Efremov, Yu. N. 1995, AJ, 110, 2757

Efremov, Yu. N. \& Elmegreen, B. G. 1998, MNRAS, 299, 588

Elias, F., Alfaro, E. J., \& Cabrera-Caño, J. 2009, MNRAS, 397, 2

Elmegreen, B. G. 2007, ApJ, 668, 1064

Elmegreen, B. G. 2008, ApJ, 672, 1006

Elmegreen, B. G. \& Elmegreen, D. M. 1987, ApJ, 320, 182

Elmegreen, B. G. \& Efremov, Yu. N. 1996, ApJ, 466, 802 
Elmegreen, B. G. \& Efremov, Yu. N. 1997, ApJ, 480, 235

Elmegreen, B. G., Elmegreen, D. M., \& Leitner, S. N. 2003, ApJ, 590, 271

Elmegreen, B. G., Elmegreen, D. M., Chandar, R., Whitmore, B., \& Regan, M. 2006, ApJ, 644, 879

Engargiola, G., Plambeck, R. L., Rosolowsky, E., \& Blitz, L. 2003, ApJS, 149, 343

Enoch, M. L., et al. 2006, ApJ, 638, 293

Fall, S. M. \& Zhang, Q. 2001, ApJ, 561, 751

Feigelson, E. D., Martin, A. L., McNeill, C. J., Broos, P. S., \& Garmire, G. P. 2009, AJ, 138, 227

Feitzinger, J. V. \& Braunsfurth, E. 1984, A\&A, 139, 104

Feitzinger, J. V. \& Galinski, T. 1987, A\&A $A, 179,249$

Gao, S., Jiang, B.-W., \& Zhao, Y.-H. 2007, ChJAA, 7, 111

Georgiev, I. Y., Hilker, M., Puzia, T. H., Goudfrooij, P., \& Baumgardt, H. 2009, MNRAS, 396, 1075

Gieles, M., Bastian, N., \& Ercolano, E. 2008, MNRAS (Letters), 391, L93

Gieles, M., Larsen, S. S., Bastian, N., \& Stein, I. T. 2006a, A\&̈A, 450, 129

Gieles, M., Larsen, S. S., \& Sheepmaker, R. A. 2006b, A\&3A (Letters), 446, L9

Gomez, M., Hartmann, L., Kenyon, S. J., \& Hewett, R. 1993, AJ, 105, 1927

Grabelsky, D. A., Cohen, R. S., Bronfman, L., Thaddeus, P., \& May, J. 1987, ApJ, 315, 122

Gutermuth, R. A., Megeath, S. T., Pipher, J. L., Williams, J. P., Allen, L. E., Myers, P. C., \& Raines, S. N. 2005, ApJ, 632, 397

Harris, W. E. 2009, ApJ, 699, 254

Harris, W. E., Whitmore, B. C., Karakla, D., Okoń, W., Baum, W. A., Hanes, D. A., \& Kavelaars, J. J. 2006, ApJ, 636, 90

Hatzidimitriou, D. \& Bhatia, R. K. 1990, A\&A, 230, 11

Hunter, D. A., Baum, W. A., O’Neil Jr., E. J., \& Lynds, R. 1996, ApJ, 456, 174

Hunter, D. A., Elmegreen, B. G., Dupuy, T. J., \& Mortonson, M. 2003, AJ, 126, 1836

Ivanov, G. R. 2005, Publ. Astron. Soc. Rudjer Boskovic, 5, 75

Johnstone, D., Wilson, C. D., Moriarty-Schieven, G., Joncas, G., Smith, G., Gregersen, E., \& Fich, M. 2000, ApJ, 545, 327

Johnstone, D., Fich, M., Mitchell, G. F., \& Moriarty-Schieven, G. 2001, ApJ, 559, 307

Jordán, A., McLaughlin, D. E., Côté, P., Ferrarese, L., Peng, E. W., Mei, S., Villegas, D., Merritt, D., Tonry, J. L., \& West, M. J. 2007, ApJS, 171, 101

Jørgensen, J. K., et al. 2006, ApJ, 645, 1246

Jørgensen, J. K., Johnstone, D., Kirk, H., \& Myers, P. C. 2007, ApJ, 656, 293

Kharchenko, N. V., Piskunov, A. E., Röser, S., Schilbach, E., \& Scholz, R.-D. 2005, A\&A, 438, 1163

Klessen, R. 2001, ApJ, 556, 837

Kundu, A., Whitmore, B. C., Sparks, W. B., Macchetto, F. D., Zepf, S. E., \& Ashman, K. M. 1999, ApJ, 513, 733

Lamers, H. J. G. L. M. \& Gieles, M. 2006, $A \mathscr{E} A$ (Letters), 455, L17

Larsen, S. S. 2009, $A \& A, 494,539$

Larson, R. B. 1995, MNRAS, 272, 213

Lynga, G. 1982, A\&\&A, 109, 213

Mackey A. D. \& Gilmore G. F., 2004, MNRAS, 355, 504

Maíz-Apellániz, J. 2001, ApJ, 563, 151

Marcolini, A., Sollima, A., D'Ercole, A., Gibson, B. K., \& Ferraro, F. R. 2007, MNRAS, 382, 443

McGee, R. X. \& Milton, J. A. 1964, Austr. J. Phys., 17, 128

McLaughlin, D. E. 2000, ApJ, 539, 618

McLaughlin, D. E. \& Fall, S. M. 2008, ApJ, 679, 1272

Megeath, S. T., Allen, L. E., Gutermuth, R. A., Pipher, J. L., Myers, P. C., Calvet, N., Hartmann, L., Muzerolle, J., \& Fazio, G. G. 2004 ApJS, 154, 367

Mieske, S. 2006, ApJ, 653, 193

Milone, A. P. 2008, ApJ, 673, 241 
Norris, J. E. 2004, ApJ (Letters), 612, L25

Odekon, M. C. 2006, AJ, 132, 1834

Odenkirchen, M., \& Grebel. E. K., 2004, in: F. Prada, D. Martínez Delgado \& T. J. Mahoney (eds.), Satellites and Tidal Streams, ASP Conf. Ser. Vol. 327, p. 284, San Francisco: ASP

Palma C., Majewski S. R., \& Johnston K. V., 2002, ApJ, 564, 736

Parmentier, G. \& Gilmore, G. F. 2007, MNRAS, 377, 352

Parmentier, G., Goodwin, S. P., Kroupa, P., \& Baumgardt, H. 2008, ApJ, 678, 347

Peng, E. W., Jordán, A., Blakeslee, J. P., Mieske, S., Côté, P., Ferrarese, L., Harris, W. E., Madrid, J. P., \& Meurer, G. R. 2009, ApJ, 703, 42

Piskunov, A. E., Kharchenko, N. V., Röser, S., Schilbach, E., \& Scholz, R.-D. 2006, A\&A, 445, 545

Rand, R. J. \& Kulkarni, S. R. 1990, ApJ (Letters), 349, L43

Rathborne, J. M., Jackson, J. M., \& Simon, R. 2006, ApJ, 641, 389

Reid, M. A. \& Wilson, C. D. 2005, ApJ, 625, 891

Sánchez, N., \& Alfaro, E. J. 2008, in: Highlights of Spanish Astrophysics $V$, in press (arXiv:0810.0478)

Sánchez, N. \& Alfaro, E. J. 2009, ApJ, 696, 2086

Scheepmaker, R. A., Lamers, H. J. G. L. M., Anders, P., \& Larsen, S. S. 2009, A $\& A, 494,81$

Schechter, P. 1976, ApJ, 203, 297

Schmeja, S., Kumar, M. S. N., \& Ferreira, B. 2008, MNRAS, 389, 1209

Smith, M. D., Gredel, R., Khanzadyan, T., \& Stanke, T. 2005, MmSAI, 76, 247

Smith, M. C., Evans, N. W., Belokurov, V., Hewett, P. C., Bramich, D. M., Gilmore, G., Irwin, M. J., Vidrih, S., \& Zucker, D. B. 2009, MNRAS, 399, 1223

Strader, J., Brodie, J. P., Spitler, L., \& Beasley, M. A. 2006, AJ, 132, 2333

Testi, L., Sargent, A. I., Olmi, L., \& Onello, J. S. 2000, ApJ (Letters), 540, L53

Ventura, P., Caloi, V., D'Antona, F., Ferguson, J., Milone, A., \& Piotto, G. 2009, MNRAS, 399, 934

Vesperini, E. 1998, MNRAS, 299, 1019

Vesperini, E. 2000, MNRAS, 318, 841

Vesperini, E., Zepf, S. E., Kundu, A., \& Ashman, K. M. 2003, ApJ, 593, 760

Waters, C. Z., Zepf, S. E., Lauer, T. R., Baltz, E. A., \& Silk, J. 2006, ApJ, 650, 885

Young, K. E., et al. 2006, ApJ, 644, 326

Zhang, Q. \& Fall, S. M. 1999, ApJ (Letters), 527, L81

Zhang, Q., Fall, S. M., \& Whitmore, B. C. 2001, ApJ, 561, 727 